\section{Mit Antidepressiva auch gegen die Negativsymptomatik}

\section{Ein hoher Anteil schizophrener Patienten wird zusätzlich zu der bestehenden antipsychotischen Medikation mit Antidepressiva behandelt. Die Empfeh- lungen der Leitlinien zu diesem zusätzlichen Gebrauch von Antidepressiva sind widersprüchlich.}

$\mathrm{n}$ der Regel werden Antidepressiva im Rahmen von Psychosen in zwei Indikationen verordnet: zum einen bei bestehender depressiver Symptomatik, zum anderen bei deutlicher Negativsymptomatik.

In der vorliegenden Metaanalyse wurden 82 Studien mit insgesamt 3.608 Teilnehmern berücksichtigt. Es handelte sich ausschließlich um sogenannte Addon-Studien, das heißt, die Antidepressiva wurden zu einer bereits bestehenden (meist) stabilen Antipsychotikamedikation hinzugegeben. Insgesamt zeigte sich ein kleiner, aber statistisch signifikanter, günstiger Effekt für die Behandlung der depressiven Symptomatik (standardized mean difference [SMD]: $-0,25,95 \%-\mathrm{KI}$ : $-0,38 ;-0,12)$, der Negativsymptomatik (SMD: $-0,30,95 \%-K I$ : $-0,44 ;-0,16$ ), der
Gesamtsymptomatik (SMD: -0,24, 95\%-KI: -0,39; -0,09), der Positivsymptomatik (SMD: $-0,17,95 \%-K I:-0,33$; $-0,01$ ), der Lebensqualität (SMD: 0,32, $95 \%$-KI: 0,57; 0,06) und der Responderrate (Risk ratio: 1,52; $95 \%$-KI: 1,29; 1,78). Der Effekt auf die depressive Symptomatik und auf die Negativsymptomatik war besonders hoch ausgeprägt, wenn diese Symptomatik eine gewisse Schwere bei den in die Studien eingeschlossenen Patienten aufwies.

\section{Kommentar}

An dieser Studie ist bemerkenswert, dass zum einen durchaus ein positiver Effekt der zusätzlichen Gabe von Antidepressiva besteht, und zwar auf die Symptomatik generell, wobei besonders erstaunlich ist, dass die Responderrate immerhin $50 \%$ höher ist unter der zusätzlichen Gabe von Antidepressiva im Vergleich zur zusätzlichen Gabe von Placebo; zum anderen gab es durchaus auch Unterschiede zwischen den Antidepressiva, insbesondere was die Behandlung der Negativsymptomatik betrifft (Abb. 1). Hier zeigt sich insbesondere Mirtazapin effektiv und auch gut untersucht (SMD immerhin -0,84, $95 \%-\mathrm{KI}:-1,31 ;-0,36)$. Der Effekt von Mirtazapin ist nur bei der Negativsymptomatik vorhanden, nicht bei der Behandlung der depressiven Symptomatik (SMD: -0,08, $95 \%-\mathrm{KI}:-0,41 ; 0,25)$. Das SNRI Duloxetin ist bei beiden Indikationen sehr effektiv (Negativsymptomatik: SMD: $-1,38$, $95 \%-\mathrm{KI}:-2,07 ;-0,68$; depressive Symptomatik: SMD: $-0,80,95 \%-K I:-1,45 ;-0,16)$, allerdings sind die entsprechenden untersuchten Patientenzahlen vergleichsweise gering (jeweils nur 40 Patienten), es liegt auch nur eine Studie vor.

Prof. Dr. med. Hans-Peter Volz

Helfer B, Samara MT, Huhn M, Klupp E, Leucht C, Zhu Y, Engel RR, Leucht S. Efficacy and Safety of Antidepressants Added to Antipsychotics for Schizophrenia: A Systematic Review and MetaAnalysis. Am J Psychiatry 2016; 173: 876-86

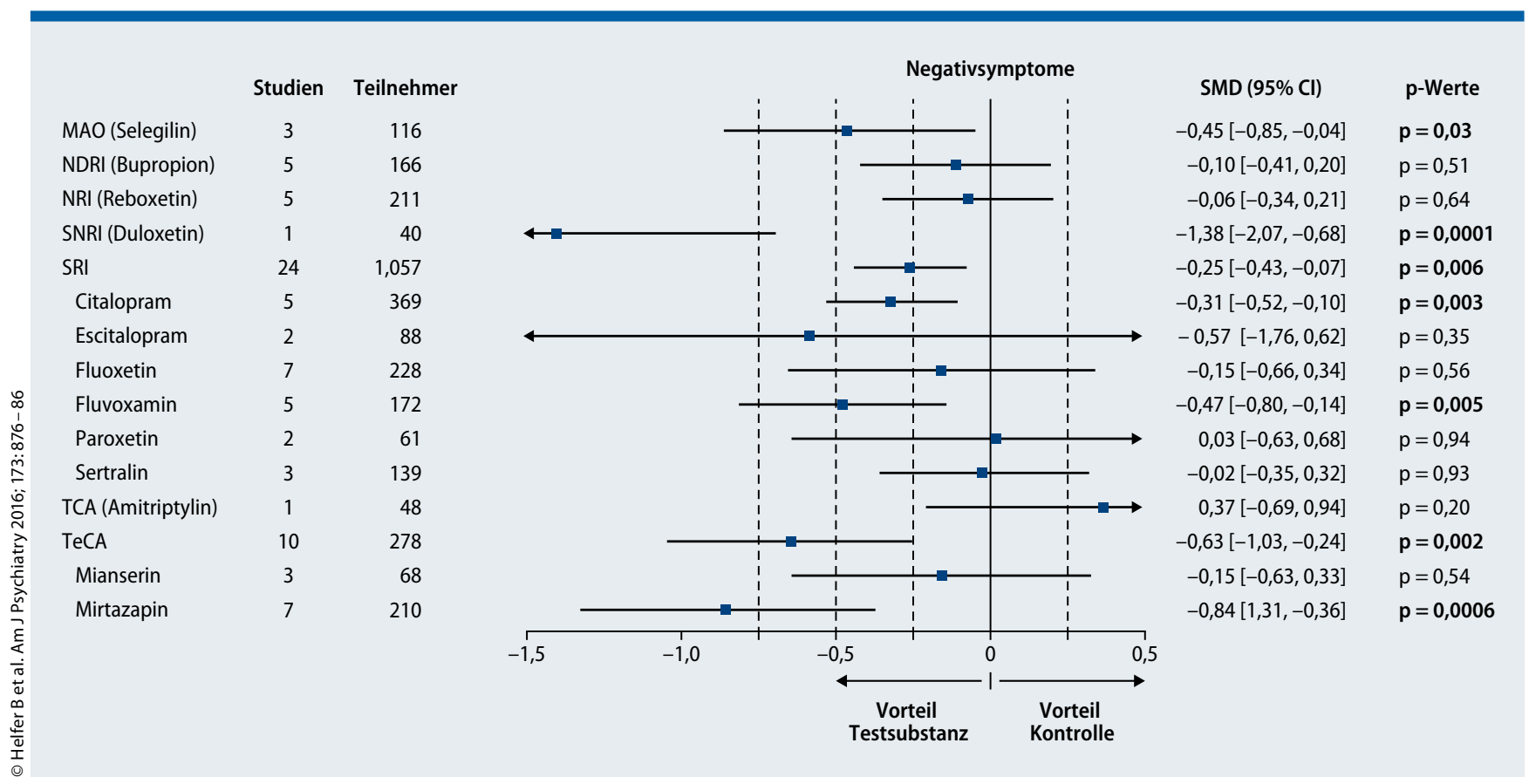

Abb. 1: Helfer et al. stellten deutliche Unterschiede zwischen den Antidepressiva fest, insbesondere was die Behandlung der Negativsymptomatik betrifft . 